\title{
A conceptual course on lasers for general education
}

\section{Mark Masters}

Mark F. Masters, "A conceptual course on lasers for general education," Proc. SPIE 9666, 11th Education and Training in Optics and Photonics Conference, 96660U (5 June 2009); doi: 10.1117/12.2208053

SDIE Event: Eleventh International Topical Meeting on Education and Training in Optics and Photonics, 2009, St. Asaph, United Kingdom 


\title{
A conceptual course on LASERs for general education
}

\author{
Mark F. Masters ${ }^{a}$ \\ Department of Physics, Indiana University Purdue University Fort Wayne \\ Fort Wayne IN 46805, USA
}

\begin{abstract}
It is important to improve the technological skills and scientific understanding of students who are not pursuing scientific and technological degrees because they are indirectly asked to support science. To be supportive, they need to be able to evaluate scientific information as portrayed by the media. The difficulty is to find a topic which will stimulate and hold their interest in science. One such topic is LASERs. LASERs hold a fascination for students. LASERs are used in a wide array of technological devices and procedures. To understand LASERs requires an understanding of light and optics; of how light interacts with matter and with the structure of matter. Therefore, a course about LASERs can entice students who typically avoid science classes, and in particular physics classes, into taking a physics class, thereby giving us the opportunity to improve their understanding of science, their critical thinking skills and developing their appreciation of basic physics. Such a course can establish a sense of confidence in these students' ability to understand.
\end{abstract}

Keywords: lasers, active learning, interactive engagement, general education, non-technical major students, nature of science

\section{GENERAL EDUCATION}

In the United States, pre-college science education tends to be weak for students with little interest in science. It is especially weak in physics which is often offered as an elective in a student's penultimate year. It is not unusual for students to enter university without any physics background at all. Additionally, student experience in science classes all too often stresses memorization and rules rather than understanding, leading to either fear or dislike of science.

U.S. universities require students to complete general education programs in addition to the studies of their major field in an effort to make up for shortcomings in the high school educational experience. As with any program, general education has its own goals and outcomes. Typically, one of the segments of general education is science and it is within this category that physics must compete with other sciences desiring to serve the same group of students. Unfortunately, this is not a level playing field since students have generally taken some biology and chemistry in high school. It is doubly hard because physics has the reputation of being a particularly difficult subject that is mathematics intensive and students have math phobias leading them to avoid physics in favor of other sciences.

One of the courses we developed for attracting the general student population into physics courses is Introduction to LASERS. It is a conceptual course with minimal mathematics but significant emphasis on reasoning and critical thinking. While students will take this course because of interest in the topic, we must endeavor to engage them with science and scientific thinking. This particular task has a number of significant hurdles. First, we must change the way students think about learning. We must convince them that science is not simply about memorizing facts but is an activity; that science is not simply jargon; that science is not simply using scientific information; that science is not dictated by the elite authority known as "the scientist".

${ }^{a}$ masters@ipfw.edu, http://users.ipfw.edu/masters 
Rather, we must endeavor to convince them that science is a method of discovery and this approach is applied throughout the entire class. Ultimately, we must teach the students to think critically about physical situations and apply what they know.

Teaching method plays a significant role in this class' success in achieving its goals. First, to convince the students that science is an activity and to change how they learn we cannot allow the students to be passive. As such, interactive engagement $(\mathrm{IE})^{1-5}$ and Studio ${ }^{6-9}$ teaching methods are used. In brief, IE is a method of teaching in which the students are "minds-on" and "hands-on" with the material. The students do not simply sit and listen to the instructor and later regurgitate the instructors' words of wisdom back in answer to canned questions. Rather the students wrestle with ideas and discuss topics in groups ${ }^{10,11}$ to develop a consensus that they can articulate during a class discussion. Ultimately, everything in the class is developed through discussion.

Studio methods involve the integration of lecture and laboratory. Laboratory activities are designed to make the students explore and discover rather than blindly follow directions. Both methodologies (IE and Studio) require students to describe what they are doing, not through a formal laboratory report parroted from the laboratory hand-out, but in their own language that their colleagues can understand. It is the use of language that is critical to student learning. Language is developed to describe common reference frames and experiences. The activities in the class, whether paper and pencil or experimental investigations, provide experiences about which the students develop a descriptive language with which to communicate.

The instructor's role is significantly different in an IE/Studio class from that of a traditional lecture class. The instructor must be affirming and not adversarial, must act as a moderator and facilitator rather than as a source of information. The instructor might give a mini-lecture, but most of the time the students are talking with each other and working with materials. The instructor has the difficult task of creating an environment in which the students feel safe and secure to make mistakes and be free to discover.

\section{INTRODUCTION TO LASERS}

The Introduction to LASERs course requires activity, discussions and writing. Most students expect classes to be devices of knowledge acquisition and focus on answer making rather than knowledge generation, usage usage and sense making. Having students recognize the difference between these is part of developing the student's "habit of mind". Activities are accordingly designed so that students have to think about and observe physical situations. However, for these activities to be successful the instructor must continuously stress that students be thoughtful and provide genuine responses.

The principle goals of the course are to develop an understanding of science and to teach students about basic physics. As such, the course outline is 1) The Nature of Science, 2) The Nature of Light and Models of Light 3) Production of Light and the Interaction of Light with Matter 4) Basic Optics 5) LASERs and 6) LASER applications. In teaching this course for the past 10 years, we have developed a number of activities, both pencil and paper and experimental, to help students gain insight into science, light, optics and laser applications. A few of these activities are described.

\section{NATURE OF SCIENCE}

It is critical that the students learn about the nature of science (NOS) to help them differentiate between science and pseudo-science, build their critical thinking skills, and have them recognize that science is an activity based upon empirical evidence. Teaching students about the nature of science (NOS) is difficult because to effectively "do" science requires significant background information and understanding. Since students in a classroom are engaged in acquiring that background, it is almost impossible for them to gain insights into scientific thought through traditional laboratory investigations. In most laboratory investigations the students simultaneously explore new concepts and phenomena, use new equipment, and use new analysis techniques in every investigation. The students are overwhelmed by novelty and adopt a strategy of "tell me what I need to know and do," a strategy with which all too many instructors are willing to facilitate by providing detailed instructions. A "cook-book" like recipe to science reinforces the students' belief that 
science is formulaic rather than discovery, creativity and critical thinking. To overcome these problems we use an activity in which the students can be involved in the process of discovery, of forming and testing hypotheses and creating theories using a knowledge domain with which they are familiar: two player games. This activity is described in a forthcoming article in The Physics Teacher ${ }^{12}$ and was inspired by a quote from The Feynman Lectures:

"We can imagine that this complicated array of moving things which constitutes "the world" is something like a great chess game being played by the gods, and we are observers of the game. We do not know what the rules of the game are; all we are allowed to do is to watch the playing. Of course, if we watch long enough, we may eventually catch on to a few of the rules. The rules of the game are what we mean by fundamental physics... (However) what we can really explain in terms of those rules is very limited, because almost all situations are so enormously complicated that we cannot follow the plays of the game using the rules, much less tell what is going to happen next. We must, therefore, limit ourselves to the more basic question of the rules of the game. If we know the rules, we consider that we "understand" the world."

In this activity the students are provided with the histories of several games played by two novice, but reasonably intelligent players. The goal for the students is to determine the rules of the game. An individual guess corresponds to a hypothesis which will be supported or refuted by the data. A collection of supported hypotheses form the rules or theory of the game. This activity serves a purpose of having everyone get used to talking with each other and discussing results in the class. It also makes it clear that a scientific theory is supported by the evidence. That changing evidence may change the theory. That one can never be absolutely certain of the end results since additional data may change that. Figure 1 is an example of this activity. It includes board, moves and pieces.

\section{Move Sequences for Scigame XXVII}

The moves in the games below were made by two novices, but reasonably intelligent, players.

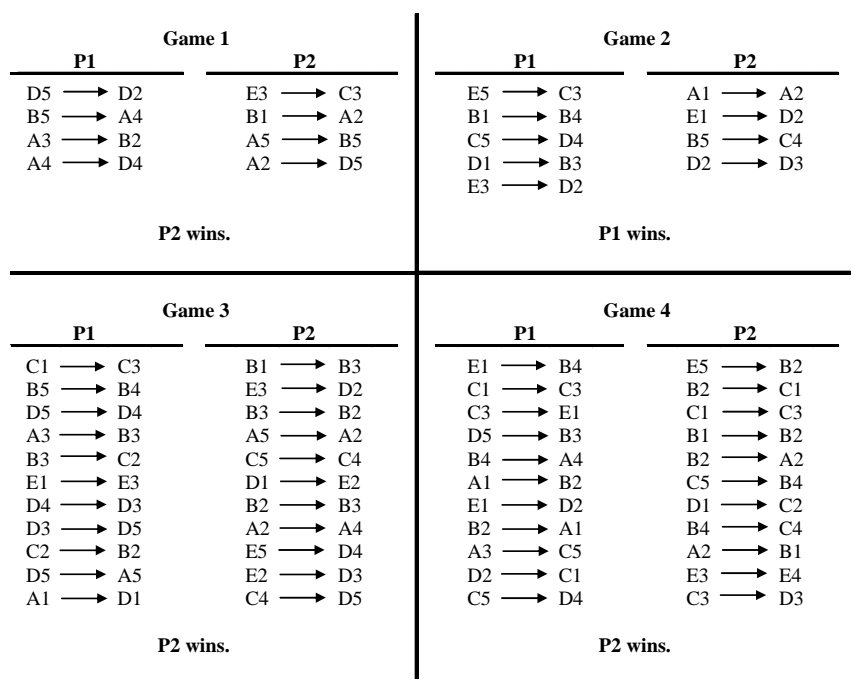

\begin{tabular}{|c|c|c|c|c|c|}
\hline & A & B & C & D & E \\
\hline 1 & & & & & \\
\hline 2 & & & & & \\
\hline 3 & & & & & \\
\hline 4 & & & & & \\
\hline 5 & & & & & \\
\hline
\end{tabular}

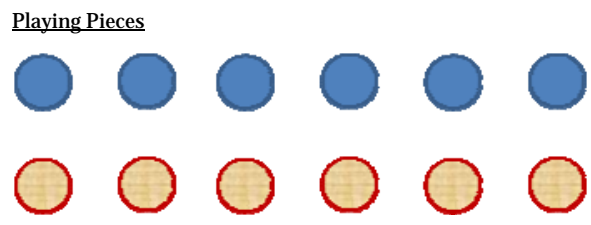

Figure 1-Game history, board and pieces for use in helping students learn some aspects of the nature of science.

The reverse game play occurs after the class has developed definitions for a scientific hypothesis and scientific theory and is used to involve the students in an analogue of scientific process rather than simply discussing it. This activity can be varied in numerous ways. For example, it is possible to provide different 
student groups with different sets of game histories (different data), none of which give them the complete game. After some time has elapsed, the groups are allowed to move around and discuss their results which emulate the social nature of constructing scientific knowledge.

The activity is generally put into context through prior discussions about the NOS. These are initiated by asking open ended questions for discussion:

- "What is the purpose of science?"

- "Does science have a basic premise?"

- "Is changeability is a sign of unreliability?"

- "Is science is a social activity"

- "Is science an activity or is it the body of knowledge?"

- "Is there a particular way to do science?"

- "What is the difference between science and engineering?"

The purpose of these questions is to get students to think about their own ideas, talk about them in a small group and write them down (which makes it easier for them to discuss in the class setting). The class discussions are such that the instructor is careful not to say whether an answer is particularly good, but to encourage the students to critique each other's answers. The instructor tabulates student responses and moderates the discussion by eliciting responses from multiple students.

A second phase of questioning asks students to consider

- Scientific terms such as hypothesis, theory and law, which the students rank in order of "closest to truth" in that order.

- Testability and proof and explanation.

- Comparing observation with explanation.

Finally, in light of the class discussions, the students are asked to determine whether a described activity is scientific or not. For example:

- "Imagine that a child constructs a model solar system using wire and painted Styrofoam balls. Has this child engaged in a scientific activity?"

The scientific structure of discovering reliable knowledge based on empirical evidence is rigorously applied throughout the remainder of the semester.

\section{NATURE OF LIGHT AND MODELS OF LIGHT}

\section{Nature of light}

To introduce "light" the class starts by considering the question "what is light?" Typically this question elicits a number of responses such as:

- "Light is moving energy,"

- "Light is energy,"

- "Light is power,"

- "Light is intense,"

- "Light is made of photons,"

- "Light is a wave"

- "Light is wavelengths"

- "Light moves in waves" or "Light travels in waves,"

- "Light is made of particles moving in waves."

The purpose of this question is not to get a correct answer; rather it is intended to make the students reflect upon what they think, and for them to hear what others think. Also, the responses, especially the ones related 
to energy give the opportunity to explore the complex and abstract concepts of work, energy and power. The core of energy discussions are based on mechanical work, kinetic energy and conservation of energy.

\section{Laser light}

While students have seen lasers, most frequently usually small laser pointers, they do not know what precisely makes the laser seem different and they are explicitly queried about the difference between laser light and ordinary light. Typical responses are:

- "Laser light is colored,"

- "it seems to move in a beam,"

- "it is powerful,"

- " "bright" or has "concentrated energy,"

- "laser light can travel further than ordinary light"

Students have little understanding of the light spectrum beyond ROYGBIV. Therefore, they have no reference for monochromaticity. Another language problem is intensity (really irradiance). Intensity is used interchangeably to mean brightness of light, vividness of color and light power. The laser beam is believed to be perfectly collimated. Finally, the students have a belief that light has a maximum distance it can travel from a source, that the light's "energy" is dissipated by traveling through the air since they believe it takes energy for something to move.

The idea of monochromaticity is not addressed until the students have started to work with the wave model of light. However, to address the latter three issues, students work through several hands-on and thought investigations. In each of these activities the students must predict what they believe will happen and then make observations and measurements to test their predictions. Finally, they must explicitly reconcile their results with their observations. Even if their predictions were correct, they must explicitly describe how the measurements support their predictions. These activities are:

A) Measurement of laser beam size with distance from laser.

B) Measurement of relative beam power and size for a lamp with a circular baffle (see Figure 2). This activity confronts the students' ideas about intensity and brightness and the dissipation of energy as light travels. The students measure the cross sectional area of the beam as the light travels farther from the baffle. A detector (photodiode) is placed in the beam at the various locations and measures the relative amount of light hitting the detector. However, since the detector is smaller than the beam of light, the detector readings diminish as it moves farther from the source. Thus, the reading varies as the apparent brightness decreases. The students are queried about why the readings decrease. Even though the students have not used lenses, they use a converging lens to concentrate the light on the detector and to observe the effect of the lens on brightness and readings. Finally, through class discussions the students develop the idea that the light is spread over a greater area, but the overall power is constant and not dissipated.
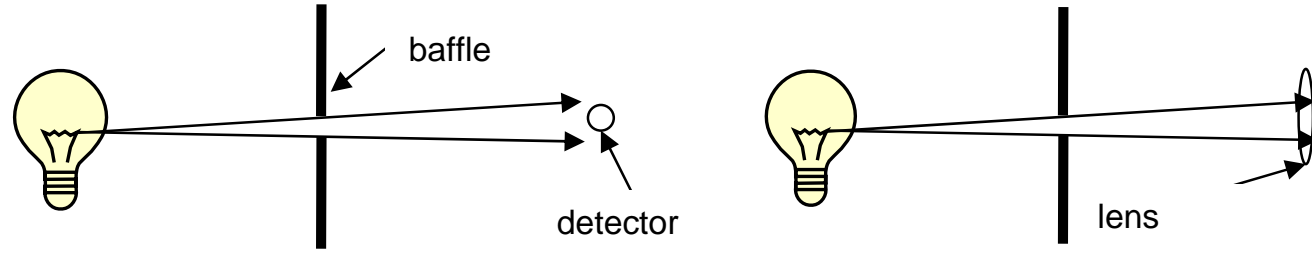

Figure 2, lamp with baffle, detector and lens 


\section{Scientific models of light: ray model}

The ray model is developed through discussions based on the following the questions:
A. What is a ray of light?
B. What information about light can a single ray indicate?
C. What would it indicate if a single ray were to end?
D. Can a single ray be subdivided?
E. What information would a collection of rays provide that a single ray could not?
F. Suppose we wanted to represent two beams of light, but one had twice the power of the other, how could you indicate this on using rays?
G. Is it possible to indicate intensity using rays? If so, how would you do so? If not, why not?

Discussions arising from these questions assist the class in interpreting ray diagrams. It is important for the students to determine what ray diagrams mean for themselves, rather than having them designed by the "authority" figure (the instructor). However, they can be led to having the relative power of a light source determined by the number of rays while the concentration of rays (how close together the rays are) determines the relative intensity. This information is used consistently throughout the semester to interpret how different optical devices function and is related to the other models. In Figure 3, the students must apply their understanding of the ray model to interpreting the ray diagrams.

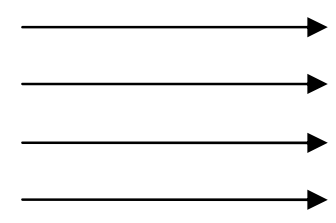

a)

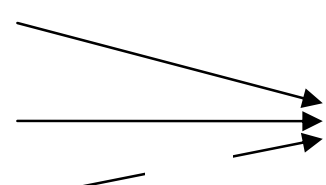

c)

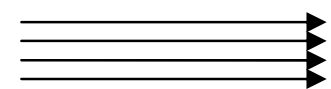

b)
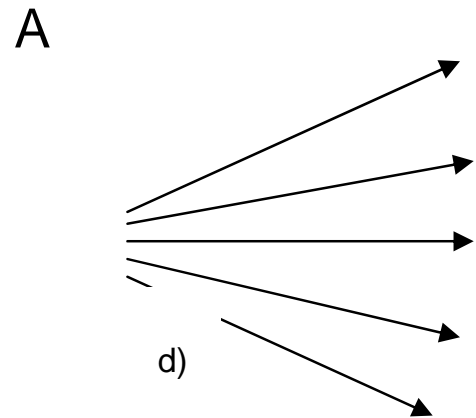

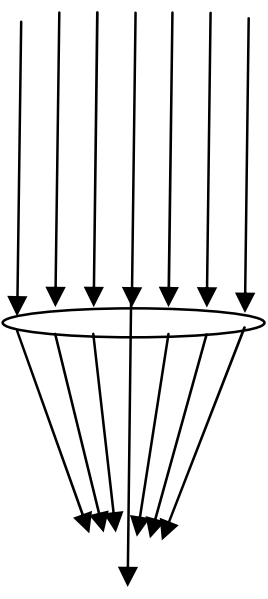

B

Figure $3-$ A) Activity using the ray model: Rank the diagrams in terms power and in terms of intensity at the arrow end of the diagrams. B) Activity helping to differentiate intensity and power. Students deduce that the light after the lens gets brighter, relating brightness and intensity, as different from power.

\section{Scientific models of light: wave model}

The wave model of light is developed to provide an explanation of certain observable light behaviors and also to use in understanding the spectrum. We educe student ideas on "what is a wave," or how do you differentiate a wave from a non-wave. You want to distinguish between what is a wave and what is not a wave. What do you believe are the properties of a wave? Three critical questions:

A. Can a wave transfer energy?

B. Can a wave transfer mass?

C. Can a wave move mass?

Students generally have the idea that the waves are only sinusoidal or that the sinusoid is the path that a wave follows. In a simple demonstration a student is asked to wake a distant sleeping student using an eraser (throwing). Clearly, in this case there is an exchange of energy and also of mass. In a second scenario, the same student is to wake the sleeping student who is advantageously holding one end of a rope. By sending a single pulse down the rope they can "jar" the student awake without transferring mass. By observing the effect of the rope pulse on the "sleeping" student, the class readily agrees that work is done on the student by the wave. The transfer of energy without the transfer of mass provides the basic definition of a wave. 
Having this basic definition of a wave the class considers repetitive waves and discusses the issue of wave path, reflecting upon the previous pulse demonstration. A standing wave is NOT used because one cannot see it traveling! The following questions are posed:

A. Imagine that light is a wave, what path does the light follow?

B. Define the following terms: wavelength, frequency, period, amplitude and speed.

C. What would happen to the frequency and wavelength of a wave if you were to double the amplitude of the wave?

In answer to (C), students often respond "That the frequency decreases if you double the wave's amplitude because for a wave on a string the speed with which the string at one location oscillates up and down is fixed. Therefore, if you increase the amplitude of the wave, it will take longer to complete one cycle, which increases the period and therefore decreases the frequency." This idea generally gives rise to many heated debates.

To help the students learn about how wavelength and frequency are related, and the effect of amplitude the students "assemble" a simple paper "wave machine" (see Figure 4). The wave machine consists of several different amplitude and wavelength sinusoidal patterns printed on strips of paper (wave-strip). A channel is made by folding a second piece of paper and fastening it with tape. The folded piece has a slot to allow one to see the "wave-strips" through the window. The window allows you to see the wave progressing with the viewer at a single location. By dragging the "wave-strip" more quickly through the envelope, one observes the frequency increasing. To measure wavelength, one has to stop the wave and use a ruler. Changing the amplitude, while keeping wave speed and wavelength constant does not change the frequency or wavelength of the wave. Finally, the students can discover that the wave travels a distance of one wavelength in one period.
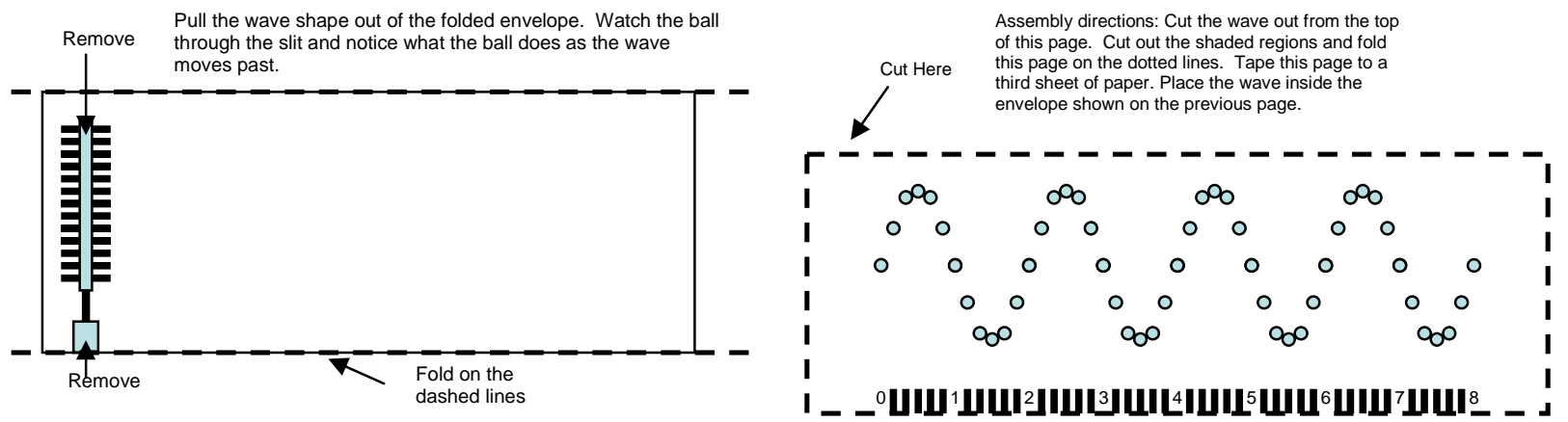

Figure 4 - "Wave machine" for relating wavelength, frequency and amplitude.

Students' are often confused by our (the instructor's) 2-D drawing of a wave, thinking that the amplitude of the wave represents the height of the wave (having units of length), and therefore the amplitude represents the spatial extent of the wave. To address this issue students relate the ray model, which describes the spatial qualities of light with the wave model through several activities shown in Figures 5 and 6.

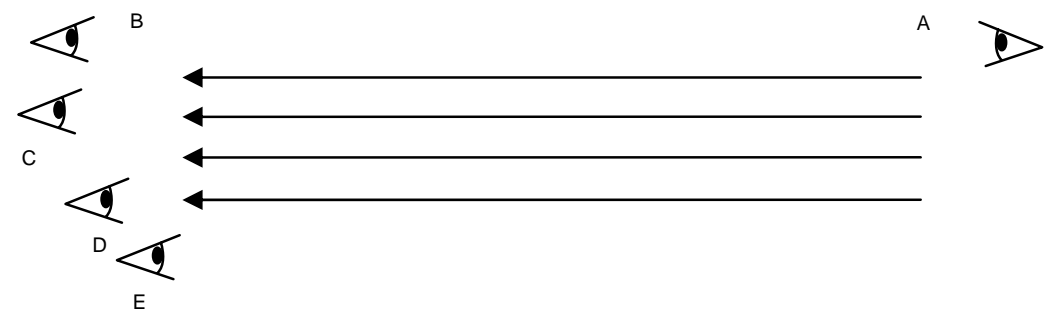

Figure 5: Which observer can see the beam of light? How do you know? How would the diagram change if you doubled the intensity of the beam? Would that change which observers could see the light? 


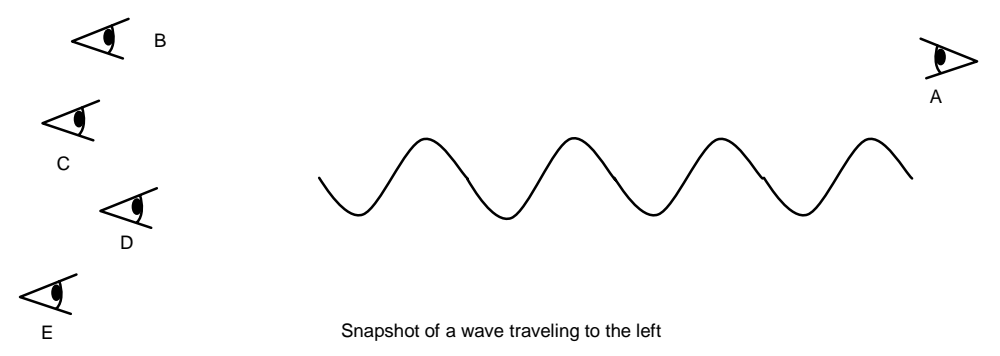

Figure 6 - In this situation, which observer can see the beam of light? How do you know? How would the diagram change if you doubled the amplitude of the beam? Would the increase in amplitude change which observers could see the light?

Using the magnifying glass ray diagram of Figure 3B and through experience of lighting paper on fire (or at least making it smoke) using a magnifying glass, the students are led to consider why the lens makes it possible to light a fire from a wave perspective. By the ray diagram the power of the light is not increased, but the intensity has increased. Through this line of questioning, the students make the connection that the amplitude of the wave is related to the intensity of the light. The students are then expected to be able to apply this to various situations as shown in Figure 7.

a)

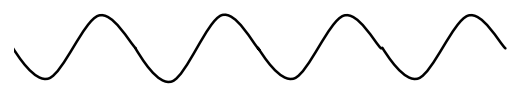

b)

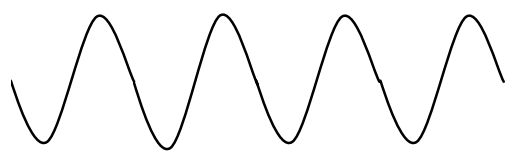

c)

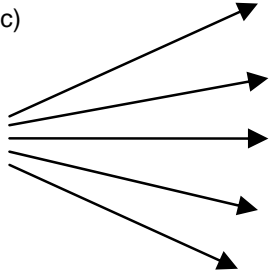

Figure 7 - Sketch a ray diagram representing as much equivalent information for the wave diagrams in a) and b) as possible. In c), sketch a wave representation of this ray diagram, presenting as much equivalent information as possible.

\section{Spectral power distributions}

The spectral power distributions (SPD) is developed to describe light's power distribution as a function of wavelength, to assist students in understanding monochromaticity, and to introduce the interaction of light with matter. The SPD is generated through student observations of a white light source using a prism. A scenario is presented in which the students imagine a small detector is moved across the spectrum, recording the "quantity of light illuminating the detector. Students consider how the chart would appear if they had only red or blue light. The students explore and refute several hypotheses: 1) the light is "colored" by the prism, and 2) the color we see is a direct property of the light. For 2), the students combine red and green light to produce yellow light and contrast this with the yellow light from the spectrum.
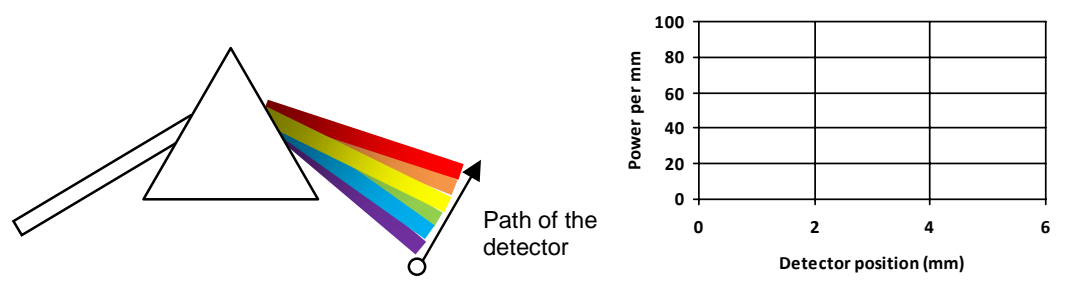

Figure 8 - Diagram of simple spectrograph for students to produce their own spectral power distribution. 


\section{INTERACTION OF LIGHT WITH MATTER AND THE PRODUCTION OF LIGHT}

The effect of the prism on white light immediately gives rise to the question: "why does the prism make light produce the different colors?" This behavior is explained through the concept of resonance which is explored for a mechanical system.

A. Students observe the effect on a pendulum bob of driving frequency, discovering that the closer one gets to the resonant frequency of the pendulum, the greater the magnitude of the pendulum swings.

B. To model how the resonance frequency would change for different systems, the students imagine that the more tightly bound the electron, the shorter the pendulum string (electron closer to the nucleus in a Bohr model) discovering that the resonance increases as the more tightly bound the electron.

Discussions reveal that prisms are made of a material with resonances in the ultraviolet portion of the spectrum and therefore light with a frequency closer to the ultraviolet portion of the spectrum will have a stronger interaction with the prism material than light of longer wavelengths. Because of this stronger interaction, the shorter wavelength light will travel more slowly, have a greater index of refraction and therefore bend at a greater angle than will longer wavelengths.

\section{Energy level diagrams}

The semi-classical resonance model works well for situations describing excitation from the ground state, but not so well to describe multiple transitions. To gain a deeper understanding of the production of light and the interaction of light with matter, we develop the concept of energy level diagrams (ELD's). However, ELD's are quite mystifying for students. One significant misconception students hold is that the "electron is excited." This is because in the Bohr model, when an atom is excited, it is indicated by an electron changing orbital.

ELD's are expanded to include molecules and condensed matter after the description that atoms cannot all have precisely the same energy levels. Since an atomic gas is characterized by very widely separated atoms that do not interact, these appear as essentially narrow energy levels. However, when there is interaction, such as in a molecule or in a liquid or a solid, the energy levels move around creating a band structure.

Using a paper and pencil activity, the students work with simplified ELD's. The students are able to construct possible emission and absorption spectra. They are expected to look at the spectra and identify the types of system (atomic, molecular or condensed). Finally, the students look at an atomic source using small spectroscopes and based on relative brightness of transitions, sketch possible energy level diagrams. While these are necessarily inaccurate, they serve the purpose of having the students think, relate and apply what they have learned. Figure 9 is an example activity in which the students must interpret the ELD, and predict a spectrum.
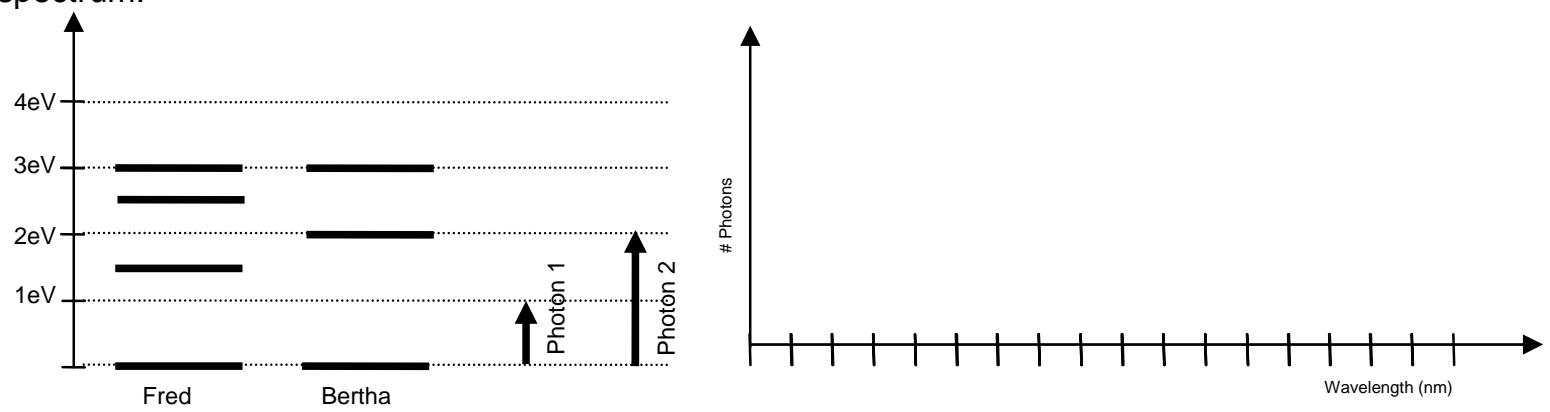

Figure 9 - Energy level diagram used by students to determine which photons interact with which atom and then determining a possible spectrum. The spectrum is determined by starting at the highest energy level shown, and then choosing every possible transition until all routes to the ground level have been determined. Then the number of photons of each energy is plotted. 


\section{BASIC OPTICS}

There are a number of activities that are used to understand basic optics. The first is for the students to discover the difference between point and extended sources through the geometric optics tutorial in McDermot's Tutorials in Physics. This is particularly important because all image formation is based on extended sources being a collection of point sources.

The laws of reflection and refraction are discovered by visual ray-tracing. Two pins are used to define an incident or exiting ray. Removing the mirror or the refracting object, the rays can be traced so that the way the ray's path can be observed. Then the formal rules of ray-tracing a point object are discussed. Rather than simply having students repeat the variety of ray-tracing activities that start with an object and end with finding the location, size and orientation of the image, we provide alternative activities, such as that in Figure 10 , that require the students to think about the meaning of images and ray-tracing.

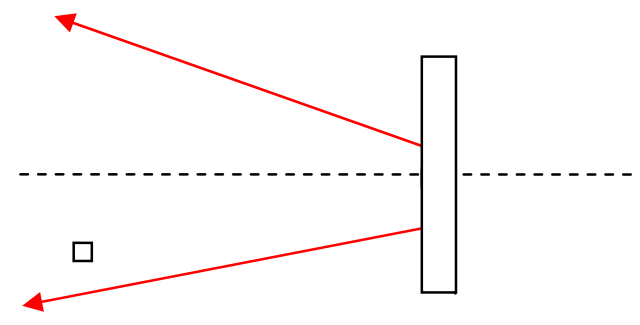

Figure 10 - Consider the situation shown below with an unknown type of mirror (plane, concave or convex) hidden by the rectangular box, a small object (the square) and two rays reflected the mirror. A) Determine the location of the image of this object. Explain. B) Determine the type of mirror. Explain your logic. C) Determine the location of the focal point of this mirror and mark it on the diagram. Explain.

\section{Interference and diffraction}

To examine interference the students predict what they would expect to see if light were to pass through a double slit, and what they would observe if the separation between the two slits were changed. Based on their observations students realize that the pattern is not simply the shadow of the slits. As part of this activity the students sort a set of wire grids in order of increasing wire separation and explain the ordering.

To explain these observations we use a model of the Michelson interferometer to develop the idea of interference being introduced through path difference. The Michelson interferometer makes the path induced phase shifts more obvious than for the double slit and allows one to ignore the difficulties associated with diffraction.
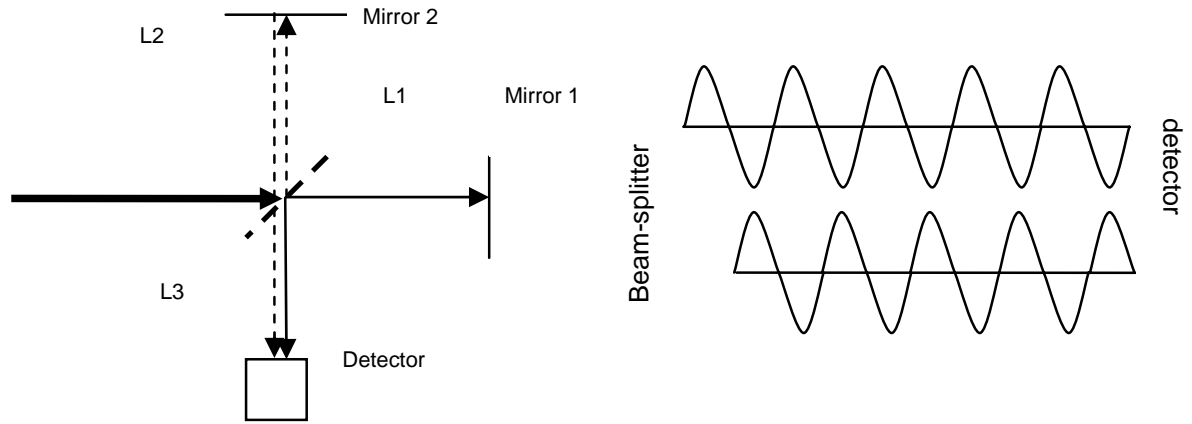

Figure 8 - On the left is a diagram of a Michelson interferometer. Directions: Assume that we have a wavelength of light that is $2 \mathrm{~cm}$ long, that $L 1=3.5 \mathrm{~cm}, L 2=3 \mathrm{~cm}$ and that $L 3$ is $4 \mathrm{~cm}$ long. A) For the light traveling to Mirror 1 or 2, what is the total path length that the light travels from the beam splitter to the detector and how long is this path in wavelengths? B) Draw lines that are the length of each path for the light to travel from the beam splitter to each mirror. On the right is the expected work showing that light would arrive out of phase at the detector. 
The students are expected to "unwrap" each of the paths the wave follows, and determine what the relative phase is for different path lengths. Using the interferometer, students can recognize that the path difference introduces a phase shift between the two beams and then they can apply the principle of superposition. They can figure out what happens as one mirror moves and gain insights into how interferometers work. This lays the ground work for understanding longitudinal coherence. Later, the students revisit the double and single slit investigations.

\section{LASERS}

Finally, having the explored so many of the intricacies of physics: models of light, spectra, production of light, optics, the students are ready to figure out how a laser functions! To start, the three basic components of a laser (medium, power source, and cavity) are discussed in general. Starting from energy level diagrams, the three photon-matter interactions (stimulated emission and absorption, and spontaneous emission) are discussed. Kinesthetic models using ping pong balls as photons are used to help the students recognize the difference between spontaneous and stimulated processes. Population inversion and lifetimes of various states are explored through a model of water reservoirs with various leak rates and pumps. In Figure 8 , an assignment is given that has the students consider what might happen to population inversions if the lower laser level of a medium has a long lifetime. The general models of three and four level laser systems are presented to the students. To make them work with these ideas the students are provided with paper and pencil assignments such as the one shown in Figure 9. They are expected to identify if a system is a 3 or 4 level laser system as in Figure 10.
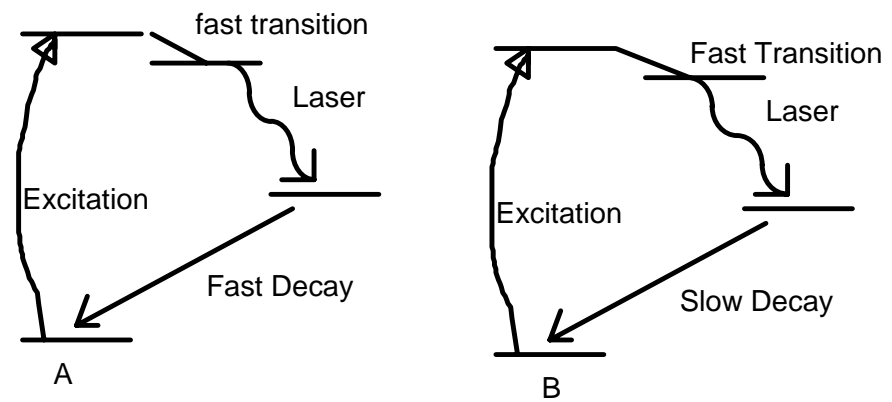

Figure 9: Consider the two systems A and B. Discuss whether a laser system based on A or B would function as a continuous or a pulsed laser.

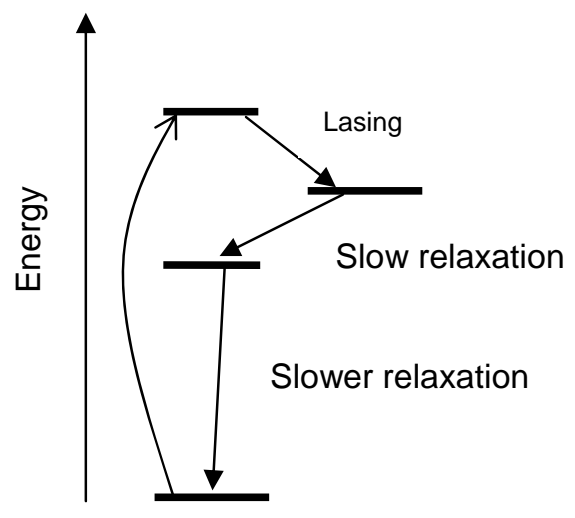

Figure 10: Does this system correspond to a three or four level laser system. 


\section{CONCLUSION}

By starting to examine the LASER, one has to teach the students a wide array of physics. The students have to learn about work and energy, light, the spectrum of light, light's interaction with matter, and the production of light before ever really considering the LASER system itself. Furthermore, the exploration of laser applications uses all of the tools developed earlier in the class such as interferometry and optics. Finally, all of this class is taught, not as a lecture, but rather as a student driven discovery based on the scientific thinking methodology with which the class started.

Even though this is a conceptual class, teaching it has given us insights into how students think about physical problems and these insights are applicable even to advanced physics student. Much of the course material we developed for this class has been modified and adopted in intermediate and advanced classes.

Finally, a text has been written in a refutational style (readers are requested to answer questions like in a discussion format class). This text and activities are available on request by email to the author.

\section{REFERENCES}

[1] R. Hake, Interactive-engagement Versus Traditional Methods: A Six-thousand Student Survey of Mechanics Test Data for Introductory Physics Courses, American Journal of Physics 66 64-74 (1998).

[2] P. Laws, Millikan Lecture 1996: Promoting active learning based physics education research in introductory physics courses, American Journal of Physics 65 14-21 (1997).

[3] L. C. McDermot, Oersted Medal Lecture 2001: "Physics Education Research - The Key to Student Learning, American Journal of Physics 69 1127-1137 (2001).

[4] L. C. McDermot and P. R. G. a. U. o. Washington, Physics by Inquiry, John Wiley and Sons, Inc., New York, 1996.

[5] E. Redish, Millikan Lecture 1998: Building a Science of Teaching Physics, American Journal of Physics $67562-$ 573 (1999).

[6] P. Laws, John Wiley and Sons, Inc., New York, 2002.

[7] E. Redish, J. Saul, and R. Steinberg, On the effectiveness of active-engagement microcomputer-based laboratories, American Journal of Physics 65 45-54 (1997).

[8] D. Sokoloff, P. Laws, and R. Thornton, RealTime Physics: active learning labs transforming the introductory laboratory, European Journal of Physics 28 S83-S94 (2007).

[9] D. Sokoloff and R. Thornton, Using Interactive Lecture Demonstrations to Create an Active Learning Environment, The Physics Teacher 35340 (1997).

[10] N. Lasry, E. Mazur, and J. Watkins, Peer Instruction: From Harvard to the two-year college, American Journal of Physics 76 1066-1069 (2008).

[11] E. Mazur, Peer Instruction: A User's Manual, Prentice Hall, Upper Saddle River, 1997.

[12] D. P. Maloney and M. F. Masters, Learning the game of Formulating and Testing Hypotheses and Theories, The Physics Teacher (expected 2009).

[13] R. Chabay and B. Sherwood, Matter \& Interactions II: Electric \& Magnetic Interactions, John Wiley \& Sons Inc., 2002.

[14] L. C. McDermot and P. Shaffer, Tutorials in Introductory Physics, Prentice Hall, 2002. 